\title{
Balance eating and exercise to prevent obesity: regularity required
}

\begin{abstract}
Obesity as a rising problem of the modern age requires meticulous prevention strategies. This article highlights the significance of a balance between eating and exercise in overcoming obesity. Increased irregular exercise can greatly increase appetite and eating and cause obesity. Care must be taken to maintain regular and moderately intense patterns of exercise to have eating under control.
\end{abstract}

Keywords: obesity, balance, eating, exercise, regularity
Special Issue - 2016

\author{
Akbar Nikkhah \\ Department of Animal Sciences, University of Zanjan, Iran
}

Correspondence: Akbar Nikkhah, Chief Highly Distinguished Professor, Department of Animal Sciences, Faculty of Agricultural Sciences, University of Zanjan, Foremost Principal Highly Distinguished Elite-Generating Scientist National Elite Foundation, Iran, Email anikkha@yahoo.com

Received: May 31, 2016 | Published: June 01, 2016

\section{Discussion}

Obesity as a disease of the modern age reduces life quality and satisfaction severely. Thus, effective prevention strategies are required to minimize its occurrence. Confusion and controversy exist on the relative importance of eating and exercise in helping to prevent obesity. This article, therefore, provides a new perspective that highlights how a delicate balance between eating and exercise can reduce obesity.

Eating and exercise must have fitting circadian patterns to target the same goal. ${ }^{1-4}$ Since eating is usually practiced on given rhythms (e.g., a minimum of three times major meal eating plus inter-meal snacks and fruits), it's fitting exercise must also occur on certain rhythms such that substrate supply and oxidation rates are harmonized..$^{5-7}$ Any uncertainty or irregularity in performing exercise may exacerbate the problem..$^{8-12}$ One obvious example is when exercise is conducted irregularly (e.g., once in a while) and over-intensely that stimulates appetite and causes overeating. The latter increases energy deposition and fuels a prolonged imbalance of substrate supply and oxidation in favour of energy supply and obesity.

Exercise does not need to be over-intense to augment weight loss and prevent obesity. As long as it is practiced regularly, intensely and joyfully, exercise can fuel a prolonged gradual balance between nutrient supply and oxidation that prevents unnecessary weight gain. An ideal strategy is to have physical activity every day and multiple times a day. However, successful strategies include exercising daily or every other daily but intensely enough to cause sweating and prolonged rises in heart rate. Having intense physical activity for only once a week would neither be sufficient nor completely healthy. Morning eating and evening exercise are additional examples in creating a healthy balance between eating and exercise. ${ }^{13}$

Perfectly, each and every eating-sitting session should have its corresponding energy-burning exercise session. ${ }^{14-17}$ The desperately busy lifestyle may not allow this to be realized. Nonetheless, what can be done are reduced eating-sitting and increased exercise to maintain a balance between nutrient supply and oxidation. ${ }^{3-5}$ For example, taking multiple small meals a day and performing at least one intense exercise session daily or every other daily help in narrowing the balance gap. Maintaining such a balance can be critical in securing a quality cancer-free lifestyle. ${ }^{2}$

\section{Implication}

Exercise must be performed regularly and frequently and not necessarily over-intensely but infrequently. Irregular and infrequent exercise can greatly increase appetite and eating and cause obesity.

\section{Acknowledgements}

Thanks to the Ministry of Science Research and Technology and National Elite Foundation for supporting the author's global initiatives and programs of optimizing science edification in the third millennium.

\section{Conflict of interest}

The author declares no conflict of interest.

\section{References}

1. Nikkhah A. Evening Exercise: A Global Strategy to Prevent Central Adiposity and Cardiometabolic Diseases. Adv Weigh Manag Obes Cont. 2015.

2. Nikkhah A. Wrecked oncogenesis through synchronized substrate availability and oxidation: A novel bioengineering of cell physiology. Austin J Biotechnol Bioeng. 2015;2(2):1042-1043.

3. Nikkhah A. Nature as an Ideal Rhythm Model for Optimal Cardiovascular physiology and Health. Int J Diabetol Vasc Dis Res. 2015;3(2e):1-2.

4. Nikkhah A. Secure Weight Management via Fitting Circadian Patterns of Physical Activity, Resting and Eating. Adv Obes Weight Manag Cont. 2015;2(4):00023.

5. Nikkhah A. Harmonizing Eating and Exercise Circadian Rhythms for Optimal Glucose-Insulin and Vascular Physiology. Int J Diabetol Vasc Dis Res. 2015;3(3):87-88.

6. Nikkhah A. Lifestyle Optimization: Today's Foremost Probiotic. J Prob Health. 2015;3:e119.

7. Nikkhah A. Improving Life Quality via Circadian Timing of Nutrient Intake: Linking Ruminant Agriculture to Human Health. EC Agriculture. 2015;2(1):258-259.

8. Nikkhah A. Meal Optimization to Reduce Obesity. Adv Obes Weight Manag Control. 2016;4(5):00105.

9. Nikkhah A. Feeding the Obese World. Adv Obes Weight Manag Control. 2016;4(5):00104. 
10. Nikkhah A. Eating Habits. Adv Obes Weight Manag Control. 2016;4(3):00091.

11. Nikkhah A. Running a pragmatic anti-cancer probiotic. J Prob Health 2016;4:e124.

12. Nikkhah A. Evening Avoidance of Large Meals alongside Evening Exercise to Improve Maternal and Child Health. Clinics Mother Child Health. 2016;13:224.

13. Nikkhah A. Morning Eating and Evening Exercise: Towards an AntiCancer Lifestyle. J Cancer Prev Curr Res. 2016;4(4):00127.
14. Nikkhah A. Nutritional Creed. Adv Obes Weight Manag Control. 2016;4(5):00103.

15. Nikkhah A. Cutting the Hunger. Adv Obes Weight Manag Control. 2016;4(1):00077.

16. Nikkhah A. Synchronized Rhythms of Exercise and Eating: A Novel Public Program to Reduce Maternal and Pediatric Diabetes. Maternal and Pediatric Nutrition Journal. 2015;1:e101.

17. Nikkhah A. Food's Art. J Nutr Health Food Eng. 2015;3(1):00099. 